\title{
Fixed points and fermion mass structure from large extra dimensions
}

\author{
S. A. Abel \\ Theory Division, CERN, CH-1211 Geneva 23, Switzerland
}

S. F. King

Department of Physics and Astronomy, University of Southampton, Southampton SO17 1BJ, United Kingdom

(Received 29 September 1998; published 8 April 1999)

\begin{abstract}
We examine the fixed point behavior of Yukawa couplings in supersymmetric theories with varying numbers of dimensions. We show that Pendleton-Ross fixed point behavior is greatly amplified in the MSSM with no extra dimensions and 4 extra $5+\overline{5}$ multiplets or the MSSM with one extra large dimension and 3 extra $5+\overline{5}$ multiplets. We also show that power law running in models with large extra dimensions can give a hierarchical set of quasi-fixed points for the Yukawa couplings in a manner which is similar to the Froggatt-Nielsen mechanism. However, we also point out the limited perturbative domain in models with power law running.
\end{abstract} [S0556-2821(99)02405-4]

PACS number(s): 12.60.Jv, 11.10.Hi, 12.10.Kt

\section{INTRODUCTION}

One interesting prospect for physics beyond the standard model is that at high energies, beyond the reach of current experiment, hidden extra dimensions open up, revealing themselves through, for example, the appearance of KaluzaKlein modes [1-3]. Above the first Kaluza-Klein threshold there would be enhancement in the effects of the renormalization group. For example, in Ref. [2] it was recently noted that consistent unification can occur at much lower scales because, by changing the classical dimension of the gauge couplings, large extra dimensions cause the (effective) renormalization group equations to run as a power law with energy scale.

In this paper we follow up a different point which was emphasized in Refs. [4, 2]; that power law running may lie behind the extraordinary hierarchies observed in the fermion mass matrices. Reference [2] presented some interesting proposals in which large extra dimensions may provide an explanation for these hierarchies and hence an alternative to the Froggatt-Nielsen mechanism. It was noted, for example, that when the effective (one loop) renormalization group equations (RGE's) are run upwards to the unification scale, the Yukawa couplings appear to have a common Landau pole. Hence it was suggested that the Landau pole could be indicative of "Yukawa unification."

Here we shall carry out a more detailed analysis of these suggestions concentrating in particular on their domain of (perturbative) validity. Generally we shall find that, if the Yukawa hierarchies are generated from scratch by the renormalization group, then perturbation theory breaks down well below the unification scale. However we shall present a case in which there is a hierarchical set of fixed points which can be calculated within perturbation theory.

Our approach is to consider the existence of quasi-fixed points (QFP's) and their domain of attraction. This is be- cause, in any scenario of Yukawa "unification," 1 at least some of the Yukawa couplings must have common Landau poles and hence, by definition, will be close to QFP's. First, in the following section, we revisit the (effective) RGE's for the gauge and Yukawa couplings for cases where different generations "feel" different numbers of large compact extra dimensions via Kaluza-Klein (KK) modes. At first sight, it does indeed appear that some rather subtle features of the renormalization group in the minimal supersymmetric standard model (MSSM) (such as fixed point behavior) could be made dominant by power law running.

In Sec. III we make a general examination of the expected fixed point behavior (both Pendleton-Ross and quasi-fixed) when we extend the MSSM by allowing different particles to feel different numbers of extra dimensions.

For the Pendleton-Ross fixed points we find that when both gauge and Yukawa couplings run as a power law (even if the powers are different), attraction to these fixed points is generally neither more nor less marked than in the MSSM because the lower unification scale exactly compensates for the enhanced running. In fact the parameters describing the properties of the Pendleton-Ross fixed point (the domain of attraction for example) can be expressed in terms of the running gauge couplings and so ultimately depend only on the strength of these couplings at the unification scale. Moreover, perturbativity limits place strong constraints on the gauge couplings, so that the allowed fixed point behavior increases as the number of extra dimensions goes down. In Sec. III we also highlight a case where the fixed point behavior is stronger than usual; when the beta function for the strong coupling is $b_{3}=1$ the domain of attraction and focusing to fixed points is greatly increased. This corresponds to

\footnotetext{
${ }^{1}$ We use this term loosely to mean a scenario in which the Yukawa hierarchy is generated by the renormalization group running alone.
} 
the MSSM with one extra large dimension and 3 extra 5 $+\overline{5}$ multiplets, or the MSSM with no extra dimensions and 4 extra $5+\overline{5}$ multiplets. In both these cases the fixed point behavior can be very strong even for quite modest gauge couplings at the GUT scale. However we find that, if perturbativity limits are satisfied, the maximum hierarchy which could be generated by Pendleton-Ross fixed points is $\sim 30$.

We also examine the running to QFP's. This is the type of behavior which was indicated in Ref. [2] where an emphasis was placed on the existence of Landau poles. For the case where we just extend the MSSM by allowing extra dimensions, we find that running to fixed points cannot generate a significant hierarchy within the perturbative regime. However we also present a model with additional singlets, in which the results are more promising. This model does have standard-model-like hierarchies in the QFP's which can be calculated within perturbation theory. We add, however, the caveat that although the hierarchical QFP's lie within the perturbative regime they should be thought of as boundary conditions to the perturbative theory. It is (currently) not possible to calculate the domain of attraction of these fixed points.

\section{RGE'S WITH EXTRA DIMENSIONS}

We first write down the MSSM RGE's in a useful matrix form which we will shortly generalize:

$$
\begin{aligned}
& 4 \pi \frac{\mathrm{d} h_{U}}{\mathrm{~d} t}=-h_{U} \cdot N_{U}-N_{Q} \cdot h_{U}-\left(N_{H_{2}}\right) h_{U} \\
& 4 \pi \frac{\mathrm{d} h_{D}}{\mathrm{~d} t}=-h_{D} \cdot N_{D}-N_{Q} \cdot h_{D}-\left(N_{H_{1}}\right) h_{D} \\
& 4 \pi \frac{\mathrm{d} h_{E}}{\mathrm{~d} t}=-h_{E} \cdot N_{E}-N_{L} \cdot h_{E}-\left(N_{H_{1}}\right) h_{E}
\end{aligned}
$$

where

$$
\begin{aligned}
& N_{H_{1}}=\left(\frac{3}{2} g_{2}^{2}+\frac{3}{10} g_{1}^{2}\right)-3 \operatorname{Tr}\left(h_{D}^{\dagger} h_{D}\right)-\operatorname{Tr}\left(h_{E}^{\dagger} h_{E}\right) \\
& N_{H_{2}}=\left(\frac{3}{2} g_{2}^{2}+\frac{3}{10} g_{1}^{2}\right)-3 \operatorname{Tr}\left(h_{U}^{\dagger} h_{U}\right) \\
& N_{Q}=\left(\frac{8}{3} g_{3}^{2}+\frac{3}{2} g_{2}^{2}+\frac{1}{30} g_{1}^{2}\right)-h_{U} h_{U}^{\dagger}-h_{D} h_{D}^{\dagger} \\
& N_{U}=\left(\frac{8}{3} g_{3}^{2}+\frac{8}{15} g_{1}^{2}\right)-2 h_{U}^{\dagger} h_{U} \\
& N_{D}=\left(\frac{8}{3} g_{3}^{2}+\frac{2}{15} g_{1}^{2}\right)-2 h_{D}^{\dagger} h_{D}
\end{aligned}
$$

$$
\begin{aligned}
& N_{L}=\left(\frac{3}{2} g_{2}^{2}+\frac{3}{10} g_{1}^{2}\right)-h_{E} h_{E}^{\dagger} \\
& N_{E}=\left(\frac{6}{5} g_{1}^{2}\right)-2 h_{E}^{\dagger} h_{E} .
\end{aligned}
$$

In these equations the parameters run logarithmically with scale;

$$
t=\frac{1}{4 \pi} \log \frac{\Lambda}{\mu} .
$$

The $N_{F}$ factors come from the wave function renormalization of the $F$ superfield corresponding to one-loop diagrams with either matter, or matter-plus-gauge particles in the loop.

In the rest of this paper we shall closely follow the models described in Ref. [2]. We shall assume that the massless fields appear only as $N=1$ supermultiplets. If they have a KK tower of $N=2$ states this is because half of the states lack a zero mode as they are odd under a $Z_{2}$ orbifolding. If they do not have a KK tower of states this is because they live, for example, at the fixed points of the orbifold or at the intersection of two branes (for more details see Ref. [2]). The $N=2$ hypermultiplets contain the usual $N=1$ part plus mirror partners with the opposite charges (so that, for example, we can write mass terms for them). The $N=2$ vectormultiplets consists of the $N=1$ vectormultiplet plus an additional $N=1$ chiral multiplet which corresponds to the longitudinal degree of freedom of the massive gauge bosons. Yukawa couplings involving the hypermultiplets are restricted in $N$ $=2$ models in order to preserve the $N=2$ supersymmetry. However we will for the moment assume that a combination of trilinear $N=2$ couplings and higher order interactions between $N=2$ hypermultiplets generates a set of Yukawa couplings at the GUT scale.

Now let us consider the contribution to beta functions coming from extra sets of $N=2$ hypermultiplets appearing in towers of $\mathrm{KK}$ modes. This is meaningful if the full (but non-renormalizable) higher dimensional theory is well approximated by a truncated model Ref. [2]. (One should bear in mind that the "RGE's" we will derive do not have the same physical interpretation as in renormalizable models, in that they express the dependence of the physical parameters on the cutoff.) For example one has to assume that higher dimensional operators do not play a significant role in the evolution of the gauge and Yukawa couplings. ${ }^{2}$

First the diagrams with matter only. These diagrams can have either one or two internal $\mathrm{KK}$ modes. If there is only one, then, each time we pass a KK threshold, the diagram contributes the same as the equivalent MSSM diagram regardless of whether the external state has, or does not have, KK modes, and independently of the KK number of the internal mode. This is because the modes which live at orbifold fixed points (or at the intersection of two branes) do not

\footnotetext{
${ }^{2}$ We would like to thank R. Rattazzi for conversations on these points.
} 
conserve KK number since translational invariance in the extra dimensions is broken. These states, since they are located at fixed points, are eigenstates of position and therefore couple with the same coefficient to all the momentum eigenstates. (Momentum is not really broken in these interactions but is absorbed by the unknown dynamics which maintains the brane or orbifold configuration.) If there are two internal $\mathrm{KK}$ modes and the external mode is the zero mode of a tower of KK states, then the diagram also contributes the same as the equivalent MSSM diagram since now KK number must be conserved at the vertex. If, however, there are two internal KK modes and the external state lives at a fixed point, then every time we pass a KK threshold we must sum over all combinations of the two internal KK modes corresponding to that threshold.

Now the diagrams with matter-plus-gauge in the loop. When we cross a KK threshold, they give the same contribution as the equivalent MSSM diagram if the matter multiplet appearing in the loop has no KK tower of states. This is because of a cancellation between diagrams involving only $N=2$ multiplets (the same cancellation that gives vanishing wave function renormalization for hypermultiplets in unbroken $N=2$ theories).

In general therefore one expects two types of contribution to the beta functions at a given threshold: those which involve a single summation and and those which involve a double summation over KK modes below that threshold. We shall denote these with a single and double tilde respectively and shall, for the moment, neglect the usual MSSM contributions. It is necessary to separate these two contributions because as we shall see they give different scale dependence in the RGE's. In an effective theory with truncated KK modes the general form of the RGE's can be expressed as

$$
4 \pi \frac{\mathrm{d} x_{i}}{\mathrm{~d} t}=x_{i} \beta_{i j} x_{j}
$$

where $x_{i}$ contains the whole set of $h_{i}^{2}$ or $g^{2}$. (Actually they can only be written like this when the Yukawa couplings are diagonal, but the discussion is the same for non-diagonal Yukawas). The beta functions are now rapidly changing with scale and to estimate this we count the number of KK mode contributions below a cut-off $\Lambda$. If each KK mode is separated by a scale $\mu_{0}$ and each adds $\widetilde{b}_{i j}$ or $\tilde{b}_{i j}$ to the beta functions, then the beta function for a given $\Lambda$ is approximately

$$
\beta_{i j}(\Lambda)=X_{\delta}\left(\frac{\Lambda}{\mu_{0}}\right)^{\delta} \widetilde{b}_{i j}+X_{\delta}^{2} \frac{(\delta !)^{2}}{(2 \delta) !}\left(\frac{\Lambda}{\mu_{0}}\right)^{2 \delta} \tilde{b}_{i j}
$$

where $\delta$ is the number of large extra dimensions, $X_{\delta}$ is volume of the unit sphere in $\delta$ dimensions,

$$
X_{\delta}=\frac{\pi^{\delta / 2}}{\Gamma(1+\delta / 2)},
$$

and where we neglect the contribution from the usual MSSM states. (We are, for the moment, assuming that all particles feel the same number of extra dimensions. When the gauge and Yukawa couplings feel different numbers of dimensions, we shall take $t_{\delta}$ to be the number of dimensions felt by the gauge couplings.) The infinitesimal solutions to the RGE's at a scale $\Lambda$ are then given by

$$
\begin{aligned}
4 \pi \delta x_{i}= & \frac{1}{4 \pi} \frac{\delta \Lambda}{\Lambda} X_{\delta}\left(\frac{\Lambda}{\mu_{0}}\right)^{\delta} x_{i} \widetilde{b}_{i j} x_{j} \\
& +\frac{1}{4 \pi} \frac{\delta \Lambda}{\Lambda} X_{\delta}^{2} \frac{(\delta !)^{2}}{(2 \delta) !}\left(\frac{\Lambda}{\mu_{0}}\right)^{2 \delta} x_{i} \tilde{b}_{i j} x_{j} \\
= & \delta t_{\delta}\left(x_{i} \widetilde{b}_{i j} x_{j}+4 \pi \delta \frac{(\delta !)^{2}}{(2 \delta) !} t_{\delta} x_{i} \tilde{b}_{i j} x_{j}\right)
\end{aligned}
$$

where we have defined

$$
t_{\delta}=\frac{X_{\delta}}{4 \pi \delta}\left(\frac{\Lambda}{\mu_{0}}\right)^{\delta}
$$

Note that when $\delta=0, t_{\delta}$ assumes the usual logarithmic form. In the limit that $\mu_{0} \ll \Lambda$ we can approximate the evolution with a power law running set of RGE's.

Gathering together all of these results, we can now write down the combined effect of the KK thresholds. We shall assume that the higgs fields have towers of KK states. In addition, to treat models with different numbers of generations having KK towers, we define

$$
\Omega=\left(\begin{array}{ccc}
a_{1} & 0 & 0 \\
0 & a_{2} & 0 \\
0 & 0 & a_{3}
\end{array}\right),
$$

where $a_{i}=0(1)$ when generation $i$ is (is not) the zero mode of a tower of KK states. The contributions to the field renormalization from the single summation diagrams are

$$
\begin{aligned}
& \widetilde{N}_{H_{1}}=-3\left[\operatorname{Tr}\left(h_{D} h_{D}^{\dagger}\right)-\operatorname{Tr}\left(h_{D} \Omega h_{D}^{\dagger} \Omega\right)\right]-\left[\operatorname{Tr}\left(h_{E} h_{E}^{\dagger}\right)\right. \\
&\left.-\operatorname{Tr}\left(h_{E} \Omega h_{E}^{\dagger} \Omega\right)\right] \\
& \widetilde{N}_{H_{2}}=-3\left[\operatorname{Tr}\left(h_{U} h_{U}^{\dagger}\right)-\operatorname{Tr}\left(h_{U} \Omega h_{U}^{\dagger} \Omega\right)\right] \\
& \widetilde{N}_{Q_{i j}}=\Omega_{i j}\left(\frac{8}{3} g_{3}^{2}+\frac{3}{2} g_{2}^{2}+\frac{1}{30} g_{1}^{2}\right)-h_{U} h_{U}^{\dagger}-h_{D} h_{D}^{\dagger} \\
&+\Omega_{U}(1-\Omega) h_{U}^{\dagger} \Omega+\Omega h_{D}(1-\Omega) h_{D}^{\dagger} \Omega \\
& \widetilde{N}_{U_{i j}}=\Omega_{i j}\left(\frac{8}{3} g_{3}^{2}+\frac{8}{15} g_{1}^{2}\right)-2 h_{U}^{\dagger} h_{U}+2 \Omega h_{U}^{\dagger}(1-\Omega) h_{U} \Omega \\
& \widetilde{N}_{D_{i j}=} \Omega_{i j}\left(\frac{8}{3} g_{3}^{2}+\frac{2}{15} g_{1}^{2}\right)-2 h_{D}^{\dagger} h_{D}+2 \Omega h_{D}^{\dagger}(1-\Omega) h_{D} \Omega
\end{aligned}
$$




$$
\begin{aligned}
& \widetilde{N}_{L_{i j}}=\Omega_{i j}\left(\frac{3}{2} g_{2}^{2}+\frac{3}{10} g_{1}^{2}\right)-h_{E} h_{E}^{\dagger}+\Omega h_{E}(1-\Omega) h_{E}^{\dagger} \Omega \\
& \widetilde{N}_{E_{i j}}=\Omega_{i j}\left(\frac{6}{5} g_{1}^{2}\right)-2 h_{E}^{\dagger} h_{E}+2 \Omega h_{E}^{\dagger}(1-\Omega) h_{E} \Omega,
\end{aligned}
$$

and the double summation contributions are

$$
\begin{aligned}
& \tilde{N}_{Q_{i j}}=-\Omega h_{U}(1-\Omega) h_{U}^{\dagger} \Omega-\Omega h_{D}(1-\Omega) h_{D}^{\dagger} \Omega \\
& \tilde{N}_{U_{i j}}=-2 \Omega h_{U}^{\dagger}(1-\Omega) h_{U} \Omega \\
& \tilde{N}_{D_{i j}}=-2 \Omega h_{D}^{\dagger}(1-\Omega) h_{D} \Omega \\
& \tilde{N}_{L_{i j}}=-\Omega h_{E}(1-\Omega) h_{E}^{\dagger} \Omega \\
& \tilde{N}_{E_{i j}}=-2 \Omega h_{E}^{\dagger}(1-\Omega) h_{E} \Omega .
\end{aligned}
$$

The RGE's can then be written

$$
\begin{aligned}
4 \pi \frac{\mathrm{d} h_{U}}{\mathrm{~d} t_{\delta}}= & -h_{U} \cdot\left(\widetilde{N}_{U}+4 \pi \delta \frac{(\delta !)^{2}}{(2 \delta) !} t_{\delta} \tilde{N}_{U}\right) \\
& -\left(\widetilde{N}_{Q}+4 \pi \delta \frac{(\delta !)^{2}}{(2 \delta) !} t_{\delta} \tilde{N}_{Q}\right) \cdot h_{U} \\
4 \pi \frac{\mathrm{d} h_{D}}{\mathrm{~d} t_{\delta}}= & -h_{D} \cdot\left(\tilde{N}_{D}+4 \pi \delta \frac{(\delta !)^{2}}{(2 \delta) !} t_{\delta} \tilde{N}_{D}\right) \\
& -\left(\widetilde{N}_{Q}+4 \pi \delta \frac{(\delta !)^{2}}{(2 \delta) !} t_{\delta} \tilde{N}_{Q}\right) \cdot h_{D} \\
4 \pi \frac{\mathrm{d} h_{E}}{\mathrm{~d} t_{\delta}}= & -h_{E} \cdot\left(\widetilde{N}_{E}+4 \pi \delta \frac{(\delta !)^{2}}{(2 \delta) !} t_{\delta} \tilde{N}_{E}\right) \\
& -\left(\tilde{N}_{L}+4 \pi \delta \frac{(\delta !)^{2}}{(2 \delta) !} t_{\delta} \tilde{N}_{L}\right) \cdot h_{E} \\
4 \pi \frac{\mathrm{d} g_{A}}{\mathrm{~d} t_{\delta}}= & \widetilde{b}_{A} g_{A}^{3},
\end{aligned}
$$

where [2]

$$
\widetilde{b}_{A}=\left(\frac{3}{5},-3,-6\right)+\left(4 \eta+n_{5+5}\right)(1,1,1) .
$$

In the above, $\eta=3-a_{1}-a_{2}-a_{3}$ counts the number of generations with KK modes and we have allowed for the possibility of $n_{5+\overline{5}}$ sets of $5+\overline{5}$ multiplets.

These RGE's describe the integrated effect of the KK thresholds. In what follows we will assume that they can be resummed in the usual way to get a better approximation than the leading log approximation used in Ref. [2]. In doing this we note that the conventional resummation of one loop Yukawa RGE's can be understood as a series of nested and one-particle-reducible field renormalization diagrams. In- specting these diagrams shows that these operators and only these appear at higher order. In particular (at this level) we never need to know about the Yukawa couplings of the mirror partners of the usual MSSM fields which appear in the hypermultiplets. We stress that "running the RGE's" has a different physical interpretation to the usual procedure in renormalizable field theory although the mathematical procedure is the same. Here the RGE's represent a summation of diagrams which give finite corrections corresponding to $\mathrm{KK}$ contributions below the cut-off $\Lambda$.

We are also assuming that the perturbation theory is still valid over the region of integration; this point will be discussed in the following section. Finally we must assume that the effect of these RGE's is almost continuous or in other words that there are many KK modes before unification. (In Ref. [2] this last approximation was shown to be valid since $t_{\delta} \sim 20$.)

\section{FIXED POINTS WITH EXTRA DIMENSIONS; THE PLOT THINS}

Before considering the full running of the flavor dependent RGE's we first discuss general renormalization group behavior with large extra dimensions. In the previous section we saw that the parameters scale as a power law with energy scale as opposed to the familiar logarithmic running seen in the MSSM. In addition, when particles feel different numbers of dimensions the power is different. This naturally leads one to suppose that scaling effects will be very strong, and that they could by themselves be responsible for the hierarchies observed in the fermion mass matrices [4,2].

When renormalization group effects are strong, the main features of the running are determined, more or less, by the presence of fixed points. There are two kinds of fixed points which are familiar from the usual MSSM; they are Pendleton-Ross fixed points (PRFP's) and quasi-fixed points (QFP's) [5-8]. The PRFP is the true fixed point in the sense that couplings are attracted towards it in the infra-red. However, in the MSSM this is not the dominant feature. In the MSSM QFP's are the dominant feature (for the top-quark Yukawa) because the top mass is relatively close to the perturbativity limit, and the QFP corresponds to the value of the Yukawa coupling when there is a Landau pole at the GUT scale.

In this section we shall give a general discussion of what form these two types of behavior take when there are large extra dimensions using a simple example which will allow us to deduce all the fixed point behavior in the more complicated cases of interest. We shall find, rather surprisingly, that PRFP behavior is, in general, not expected to be a significant factor. Of course, when compared to the energy scale, the running to the PRFP is indeed very strong since in $4+\delta$ dimensions the Yukawa couplings have classical dimension. So, however, do the gauge couplings (unless the they feel none of the extra dimensions or the contributions to their beta functions vanish) and it is these that set the scale of unification. It is not likely therefore, that PRFP's play a large part in the generation of fermion hierarchies. We also find that QFP's are not expected to be significant if the only 
extension we make to the MSSM is to allow some particles to feel extra dimensions. A number of other scenarios can have hierarchical QFP's however, and we will examine one case which involves extra singlets.

\section{A. PRFP's in a generic case}

To be more specific, let us examine the renormalization of a Yukawa coupling, $h_{t}$ (which, for the sake of argument, we shall call the top quark Yukawa), whose RGE is given by

$$
4 \pi \frac{\mathrm{d} h_{t}}{\mathrm{~d} t_{\delta}}=h_{t}\left(a h_{t}^{2}-c_{A} g_{A}^{2}\right) .
$$

If we put $a=3$ and $c_{A}=(17 / 30,3 / 2,16 / 3)$ then this is the $h_{U_{33}}$ RGE of the previous section when $h_{U_{33}}$ is dominant. This equation is also of the same form as that in the usual $\operatorname{MSSM}(\delta=0)$ except in that case $a=6$ and $c_{A}$ $=(13 / 15,3,16 / 3)$, and so we can use the same solutions. Defining

$$
\begin{aligned}
& r_{A}=\alpha_{0} / \alpha_{A} \\
& R_{t}=\frac{h_{t}^{2}}{g_{3}^{2}},
\end{aligned}
$$

where we use subscript- 0 to denote values at the unification scale, we find the solutions

$$
\begin{aligned}
& r_{A}=1-2 \widetilde{b}_{A} \alpha_{0} \Delta t_{\delta} \\
& \frac{1}{R_{t}}=\frac{1}{r_{3} R_{0} \Pi}-J
\end{aligned}
$$

where

$$
\begin{aligned}
\Pi\left(r_{A}\right) & =\prod_{A} r_{A}^{c_{A} / \tilde{b}_{A}} \\
J & =\frac{a}{\widetilde{b}_{3} r_{3} \Pi} \int_{r_{3}}^{1} \Pi \mathrm{d} r_{3}^{\prime} .
\end{aligned}
$$

To discuss the fixed point behavior it helps to define an "instantaneous" fixed point, $R_{t}^{*}(r)$, which can be thought of as the value of $R_{t}$ which is approached when the gauge couplings are renormalizing very slowly. That is

$$
\frac{1}{R_{t}^{*}}=\frac{1}{r_{3} R_{t}^{*} \Pi}-J
$$

or

$$
R_{t}^{*}=\frac{1}{J}\left(\frac{1}{r_{3} \Pi}-1\right)
$$

Substituting into $R_{t}$ gives

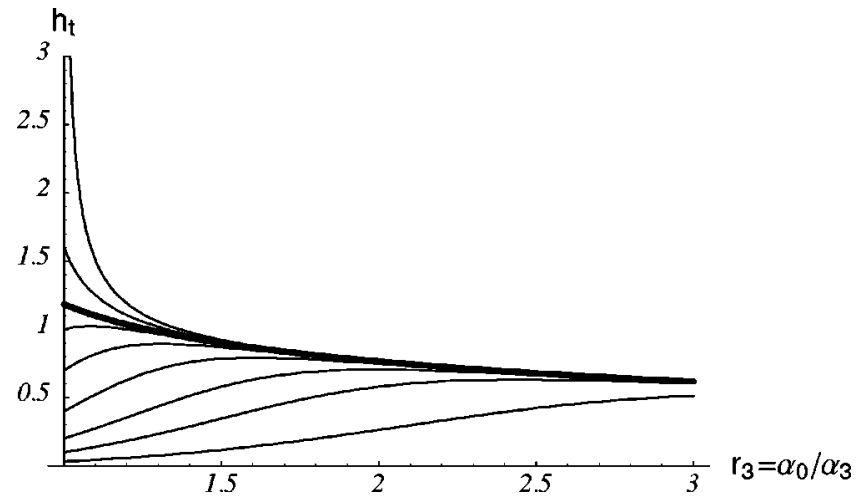

FIG. 1. Renormalization of the top quark Yukawa in the MSSM with $4 \times(5+\overline{5})$ multiplets. The bold line shows $R_{t}^{*}\left(r_{3}\right)$. Its infrared limit, $R_{t}^{*}(\infty)$, corresponds to the Pendleton-Ross fixed point. The highest line corresponds to the quasi-fixed point of Hill. Two loop effects do not change the diagram significantly. The lowest line corresponds to a $h_{t}\left(M_{G U T}\right)=0.03$.

$$
R_{t}=\frac{R_{t}^{*}}{1+\Delta\left(\frac{R_{t}^{*}}{R_{0}}-1\right)}
$$

where

$$
\Delta\left(r_{3}\right)=\frac{1}{r_{3} \Pi}
$$

defines the domain of attraction of the fixed point. In the usual MSSM $\Delta \approx r_{3}^{7 / 9} \approx 0.37^{7 / 9} \approx 1 / 2$.

The virtue of $R_{t}^{*}\left(r_{3}\right)$ is that it separates the minor effects of gauge renormalization from the "PRFP behavior" which is given by $\Delta$. [We emphasize that this is not a new kind of fixed point. For example, if the gauge coupling renormalization is as fast as that of the Yukawas then, even if $R_{t}$ is set to be $R_{t}^{*}\left(r_{3}\right)$ at some scale, it will leave the $R_{t}^{*}\left(r_{3}\right)$ line.]

The PRFP corresponds to taking the infra-red limit (i.e., $r_{3} \rightarrow \infty$ for positive $b_{3}$ or $r_{3} \rightarrow 0$ in the MSSM) to find $1 /\left(r_{3} \Pi\right) \rightarrow 0$ to give, for example,

$$
R_{t}^{*}(0)=-1 / J(0)=7 / 18
$$

in the MSSM. Here we can identify some interesting new cases where the domain of attraction is very large. For example, we can add $4 \times(5+\overline{5})$ multiplets to the MSSM to make $\widetilde{b}_{3}=+1$. In this case we find $R_{t}^{*}(0)=19 / 18$ and

$$
\Delta \approx r_{3}^{-19 / 3} \text {. }
$$

Since positive $\widetilde{b}_{3}$ means stronger coupling at unification, $r_{3}$ can be 3 for example. This gives a very large domain of attraction since now $\Delta \approx 10^{-3}$ thanks to the large power appearing above. In fact $\widetilde{b}_{3}=+1$ appears to be the optimum case. The top quark Yukawa is, in this instance, rapidly focussed (from above and below) to the running value given by $R_{t}^{*}\left(r_{3}\right)$ in Eq. (18) as shown in Fig. 1. (This behavior is unchanged by two loop corrections.) In models with one 


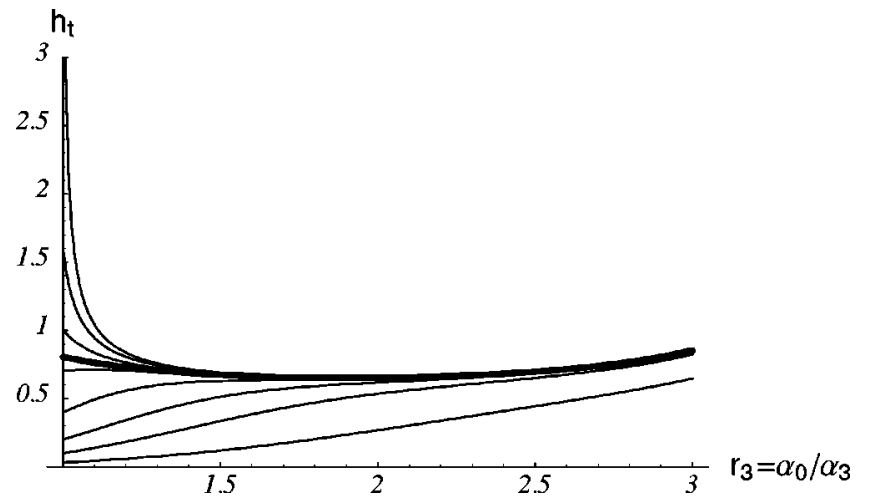

FIG. 2. Renormalization of a Yukawa coupling with double power law scaling with one extra dimension and $3 \times(5+\overline{5})$ multiplets. We choose $2 \alpha_{0} \widetilde{b}_{3}=1$ and $t_{\delta}\left(M_{G U T}\right)=2+1 / 2 \pi$ (so that $r_{3}$ $=3$ corresponds to $\Lambda=\mu_{0}$ ).

large extra dimension the equivalent would be to have one generation with KK modes and 3 additional $(5+\overline{5})$ multiplets. Then, for a dominant third generation, we still have Eq. (23) with $R_{t}^{*}(0)=19 / 9$, and we conclude that in this case the extra dimensions have not increased the running to PRFP's.

What about the cases where there are additional powers of $t_{\delta}$ in the RGE's or when, as in the RGE's of the previous section, there are double summations over KK modes? Even in these cases (even more counter-intuitively) the fixed point behavior is not particularly enhanced. Indeed when we solve the general equation,

$$
4 \pi \frac{\mathrm{d} h_{t}}{\mathrm{~d} t_{\delta}}=h_{t}\left(a\left(t_{\delta}\right) h_{t}^{2}-c_{A} g_{A}^{2}\right),
$$

where now $a\left(t_{\delta}\right)$ is any function of $t_{\delta}$, we find a solution given by Eqs. (19), (20), (21) with $J$ replaced by

$$
J_{a}=\frac{1}{\widetilde{b}_{3} r_{3} \Pi} \int_{r_{3}}^{1} a\left(t_{\delta}\left(r_{3}^{\prime}\right)\right) \Pi \mathrm{d} r_{3}^{\prime}
$$

where

$$
t_{\delta}\left(r_{3}\right)=t_{\delta}\left(M_{G U T}\right)+\frac{1-r_{3}}{2 \widetilde{b}_{3} \alpha_{0}} .
$$

Hence, only $R_{t}^{*}\left(r_{3}\right)$ is changed by any extra powers of $t_{\delta}$ and the domain of attraction, $\Delta\left(r_{3}\right)$, remains the same. Moreover it is not possible to consistently have a hierarchical set of PRFP's within perturbation theory.

Let us demonstrate the implications for a simple example. For the terms coming from double summations over KK modes in the previous section, there is a doubled power of $t_{\delta}$. We plot the resulting renormalization [with $a\left(t_{\delta}\right)=6 t_{\delta}$ ] in Fig. 2 for the MSSM with a single extra dimension and 3 additional $(5+\overline{5})$ multiplets. The extra power has not increased the fixed point behavior significantly. [We stop the renormalization at low scale at the mass of the lightest $\mathrm{KK}$ mode $\left(\Lambda=\mu_{0}\right)$.]
From the above discussion we conclude that large extra dimensions do not generically lead to enhanced PRFP behavior. The power law running to PRFP's is accompanied by an equally rapid running of the gauge couplings so that the net result is merely that unification (or loss of perturbativity) is reached at a much lower energy scale. We also see that PRFP behavior is a dominant feature of strong unification (larger $r_{3}$ gives stronger attraction) $[4,9,10]$. In models with extra dimensions the PRFP behavior is therefore ultimately limited by perturbativity constraints. Generally we require that $n$ +1 -loop diagrams contribute less than $n$-loop diagrams, which gives [10]

$$
N \widetilde{b}_{3} \alpha_{0} \lesssim 4 \pi,
$$

where $N$ is the number of degrees of freedom contributing to the $\beta$-function. In this case

$$
N=X_{\delta}\left(\frac{\Lambda}{\mu_{0}}\right)^{\delta}
$$

Perturbativity then requires

$$
\widetilde{b}_{3} \delta \alpha_{0}\left|\Delta t_{\delta}\right| \lesssim 1
$$

and hence

$$
r_{3}=1-2 \widetilde{b}_{3} \alpha_{0} \Delta t_{\delta} \lesssim 1+2 / \delta .
$$

So $r_{3} \approx 3$ is the perturbativity limit for $\delta>0$. On these quite general grounds therefore, it seems unlikely that one could generate a hierarchy of greater than $3^{19 / 6} \approx 30$ from the effects of PRFP's alone.

\section{B. QFP induced hierarchies}

Now we turn to QFP behavior which happens in an entirely different region of parameter space. Here we shall find that hierarchical QFP's can exist within perturbation theory. The QFP corresponds to taking $R_{0} \rightarrow \infty$ in Eq. (20) and hence,

$$
R_{t}^{Q F P} \approx \frac{R_{t}^{*}(0)}{1-\Delta\left(r_{3}\right)} .
$$

Now consider, simply as an example, what happens when the gauge couplings feel none of the extra dimensions but the Yukawa coupling does? This could occur if there were extra multiplets in the KK levels so that the contributions to the gauge beta functions come from complete $N=4$ multiplets and hence cancel, but the Yukawa couplings still receive contributions from towers of $N=2$ multiplets. (Since this is just an example we shall not go to the trouble of actually building such a model.) Here one would expect power law running to have a significant impact and indeed it does. In this case we can work with the usual logarithmic variable, $t_{\delta} \equiv t$, and put

$$
a\left(t\left(r_{3}\right)\right) \approx X_{\delta}\left(\frac{M_{G U T}}{\mu_{0}}\right)^{\delta} e^{16 \pi \delta\left(r_{3}-1\right)},
$$




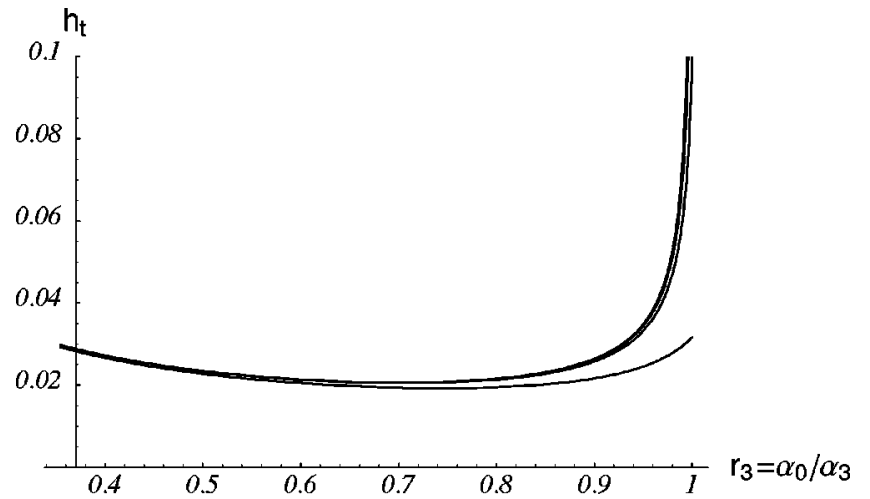

FIG. 3. Renormalization of a Yukawa coupling which feels 4 extra dimensions of $N=2$ multiplets when the gauge couplings feel only one extra dimension. We choose $\alpha_{0}=1 / 24$ and the same starting values for the Yukawa coupling as in Figs. 1 and 2.

where we have taken $\alpha_{0}=1 / 24$. The prefactor means that

$$
J_{a} \approx-\frac{3}{16 \pi \delta}\left(\frac{M_{G U T}}{\mu_{0}}\right)^{\delta} \Delta\left(r_{3}\right)
$$

is potentially huge. Temporarily ignoring the question of perturbativity, Eq. (16) tells us that

$$
R_{t}^{-1}=\Delta\left(r_{3}\right)\left(R_{0}^{-1}+\frac{3}{16 \pi \delta}\left(\frac{M_{G U T}}{\mu_{0}}\right)^{\delta}\right) .
$$

All of the the solutions are "pinched" to very small values by the QFP solution near the grand unified theory (GUT) scale. Deviation from the QFP is usually expressed with the parameter $\rho=R_{t} / R_{t}^{Q F P}$. Here we find

$$
\rho \approx 1-\frac{16 \pi \delta}{3 R_{0}}\left(\frac{M_{G U T}}{\mu_{0}}\right)^{-\delta} .
$$

So that, unless $R_{0}$ is extremely small, the Yukawa couplings effectively follow the quasi-fixed point solution.

We can see that QFP's will be important whenever the factor, $a\left(t_{\delta}\right)$ is large during the renormalization. For power law running we might hope to be able to generate hierarchies if, for example, $a\left(t_{\delta}\right)=t_{\delta}^{n}$; by examining $J_{a}$ above we find

$$
h_{t}=g_{3} \sqrt{R_{t}} \sim 1 / \sqrt{t_{\delta}^{n}\left(M_{G U T}\right)} .
$$

In Fig. 3 we show the running for the Yukawa couplings when $\delta=1, t_{\delta}=10, a\left(t_{\delta}\right)=6 t_{\delta}^{3}$, and with the same values of $c_{A}$ as in Fig. 2. The GUT scale values for the Yukawa couplings are the same as in Figs. 1, 2, so in this case it appears that hierarchies of $10^{2}$ in the Yukawa couplings have been generated. Note that when the gauge couplings are running as a power law we must respect the perturbativity constraints so that QFP's are generally more important when $\alpha_{0}$ is small and we have weak unification.

Perturbativity constraints also apply to the Yukawa couplings, so that it might appear that there is some contradiction in having such large coefficients for the Yukawa couplings in the RGE's. As for the gauge couplings we should require that

$$
t_{\delta} a\left(t_{\delta}\right) h_{t}^{2} \lesssim 4 \pi
$$

However the rough QFP values in Eq. (36) satisfy this bound if $t_{\delta} \lesssim 4 \pi$. Hence the QFP solutions should be thought of as boundary conditions. Once the model has dropped into the perturbative regime the Yukawa couplings will run to these fixed points, however we cannot explain why the model drops into the perturbative regime in the first place (although we shall make some additional comments about this in the discussion).

Now let us consider what models may exhibit such large $a\left(t_{\delta}\right)$. The RGE's we derived in the previous section were for cases in which all particles feel the same number of extra dimensions. Here the largest terms are the double summation terms with $a\left(t_{\delta}\right) \sim t_{\delta}$. Given the values of $t_{\delta}\left(M_{G U T}\right)$ $(\lesssim 20)$ found in Ref. [2], it is not possible to generate the required hierarchies unless the Yukawa couplings somehow feel more dimensions. This seems to be an unavoidable condition for generating large hierarchies from power law running.

It does not seem possible to achieve this simply by having the particles feel different numbers of dimensions (at least within perturbation theory). For example, one might try having the gauge particles feel $\delta$ dimensions and the matter and Higgs particles feel $\delta^{\prime}>\delta$ dimensions. In this case we modify the Yukawa RGE's by multiplying all of the Yukawa terms in Eqs. (10), (11) by

$$
\frac{\mathrm{d} t_{\delta^{\prime}}}{\mathrm{d} t_{\delta}}=\frac{t_{\delta^{\prime}} \delta^{\prime}}{t_{\delta} \delta}
$$

where

$$
t_{\delta^{\prime}}=\frac{X_{\delta^{\prime}}}{4 \pi \delta^{\prime}}\left(\frac{4 \pi \delta t_{\delta}}{X_{\delta}}\right)^{\delta^{\prime} / \delta}
$$

and by replacing $\delta$ by $\delta^{\prime}$ on the right-hand side (RHS) of Eq. (12), and $t_{\delta}$ by $t_{\delta}^{\prime}$. However the gauge beta functions receive contributions from all $\delta^{\prime}$ dimensions of matter and Higgs particles; hence we should also multiply $\eta$ by $t_{\delta^{\prime}} \delta^{\prime} / t_{\delta} \delta$ and thus the gauge couplings become strong before unification. In this case our previous perturbativity limits on the gauge couplings restrict $t_{\delta^{\prime}} \lesssim 1$ and prevent it from generating a significant hierarchy.

There are three possible ways we can allow the beta functions of the Yukawa couplings to feel more dimensions of KK modes; let the gauge particles interact with additional (vector and chiral) multiplets so that they feel entire $N=4$ multiplets rather than $N=2$ hypermultiplets from at least some of the extra dimensions; introduce additional nonperturbative gauge couplings for some of the fermions; modify the Yukawa couplings themselves by adding extra singlets which are fully dynamical fields above the scale $\mu_{0}$ [2]. We shall now examine the last idea (although much of the discussion will apply to the other two).

We begin with a model in which none of the matter multiplets feel extra dimensions (the $\eta=0$ scenario of Ref. [2]) 
and the Higgs and gauge multiplets feel one ( $\delta=1)$. We add two singlets, which we call $\Phi$ and $\Phi$, so that the Yukawa couplings are of the form

$$
W=h_{F_{i j}}\left(\frac{\Phi}{\mu_{0}}\right)^{n_{F_{i}}}\left(\frac{\bar{\Phi}}{\mu_{0}}\right)^{n_{\bar{F}}}{ }_{F_{i}} \bar{F}_{j} H_{u, d}
$$

We shall choose the $n_{F}$ 's and $n_{F}^{-}$'s to be functions of the generation numbers of the multiplets $F$ to introduce the required flavor dependence in the evolution of the couplings. Above the scale $\mu_{0}$ the wave function renormalization diagrams are replaced by diagrams with $n_{F}+n_{F}^{-}$extra loops of $\Phi$ and $\Phi$ particles.

The wave function renormalization receives contributions which scale as $\left(\Lambda / \mu_{0}\right)^{3\left(n_{F}+n_{F}^{-}\right)+1}$ [2] so that the RGE's of Eq. (12) are modified by multiplying the Yukawa terms by an additional factor

$$
t_{\delta}^{3\left(n_{F_{i}}+n_{F_{j}}\right)}
$$

where now $n_{F_{i}}+n_{F_{j}}$ counts the number of singlets appearing in the leading diagram. For example, let $n_{F_{i}}$ and $n_{F_{j}}$ be simply $3-i$ and $3-j$. To modify the RGE's we define

$$
\eta_{i}=t_{\delta}^{3(3-i)}
$$

In the wave function renormalization diagrams for the matter fields we then replace

$$
\begin{aligned}
& \left(h_{U} h_{U}^{\dagger}\right)_{i j} \rightarrow \delta_{i j} \eta_{i} \sum_{k} h_{U_{i k}} \eta_{k}\left(h_{U}^{\dagger}\right)_{k i} \\
& \left(h_{U}^{\dagger} h_{U}\right)_{i j} \rightarrow \delta_{i j} \eta_{i} \sum_{k}\left(h_{U}^{\dagger}\right)_{i k} \eta_{k} h_{U_{k i}}
\end{aligned}
$$

and similar for the down and lepton fields. In addition the Higgs, $\Phi$ and $\Phi$ renormalizations are suppressed by one power of $t_{\delta}$ (since the matter multiplets do not have a tower of KK excitations) so we can safely neglect them. We now resum the RGE's in the usual way (again bearing in mind that the resummation is merely a convenient way of including finite threshold effects).

We can estimate the QFP's as follows. Assume that initially the $h_{U}$ Yukawa couplings are all roughly the same size at the GUT scale and take $t_{\delta}\left(M_{G U T}\right)$ to be large (as it has to be in order to generate a hierarchy at all). We then approximate the RGE's using $\eta_{1} \gg \eta_{2} \gg \eta_{3}$. The $h_{U_{11}}$ RGE takes the same form as Eq. (14) with

$$
a_{11}\left(t_{\delta}\right) \approx 3 \eta_{1}^{2}
$$

Its low-scale quasi-fixed value is $h_{U_{11}} \sim 1 / \eta_{1}$. When $h_{U_{11}}$ is near its quasi-fixed value, the RGE for $h_{U_{i 1}}$ takes the same form as Eq. (14) with

$$
a_{1 i}\left(t_{\delta}\right) \approx 3 \eta_{1} \eta_{i}
$$

so that its quasi-fixed value is $h_{U_{1 i}} \sim 1 / \sqrt{\eta_{1} \eta_{i}}$, and so on. The final form for the quasi-fixed $h_{U}$ is found to be

$$
h_{U_{i j}} \sim 1 / \sqrt{\eta_{i} \eta_{j}}=\left(\begin{array}{ccc}
\epsilon^{4} & \epsilon^{3} & \epsilon^{2} \\
\epsilon^{3} & \epsilon^{2} & \epsilon \\
\epsilon^{2} & \epsilon & 1
\end{array}\right)
$$

where

$$
\epsilon \sim t_{\delta}\left(M_{G U T}\right)^{-3 / 2} .
$$

The final hierarchy is

$$
\left(h_{t}, h_{c}, h_{u}\right) \sim\left(1, \epsilon^{2}, \epsilon^{4}\right)
$$

and the CKM matrix is of the form

$$
K \sim\left(\begin{array}{ccc}
1 & \epsilon & \epsilon^{2} \\
\epsilon & 1 & \epsilon \\
\epsilon^{2} & \epsilon & 1
\end{array}\right) .
$$

With this ansatz the QFP's assume a structure with hierarchies similar to those in the standard model if $\epsilon \sim 0.1$ and clearly, in this case, the structure with singlets is similar to that one would have with the usual Froggatt-Nielsen mechanism. However this is also true for two examples we have not considered that do not have additional singlets. Note that from the mass hierarchies we generally expect to get an estimate of the scale $\left(\mu_{0}\right)$ at which new KK states will appear; since $\epsilon \sim 0.1$ we find $t_{\delta} \sim 10^{2 / 3} \approx 5$ and hence $M_{G U T} \sim 15 \mu_{0}$ for $\delta=1$. Consulting Ref. [2] we see that this implies

$$
\mu_{0} \sim 10^{7} \mathrm{GeV}
$$

The perturbativity discussion of the preceding section can be carried over directly to the present case and now, unfortunately, we again find that the (low scale) QFP's are close to the naive perturbative limit as is also the case for the two examples we did not consider. Perturbation theory does not allow us to follow the renormalization of the Yukawa couplings very far from their low scale values.

\section{DISCUSSION}

In this study we discussed various aspects of fixed point behavior in theories both with and without extra dimensions. We found that in models where the strong gauge beta function is $b_{3}=+1$ (the MSSM with no extra dimensions and 4 extra $5+\overline{5}$ multiplets or the MSSM with one extra large dimension and 3 extra $5+\overline{5}$ multiplets) the effects of Pendleton-Ross fixed points are greatly enhanced. However we argued that the maximum hierarchies that can be generated from Pendleton-Ross fixed points within perturbation theory are $\sim 30$.

We also examined the effect of successive KK thresholds on the running of Yukawa couplings to QFP's. We find that adding extra dimensions can enhance the effects of these fixed points appreciably; QFP's can be a dominant feature of the one loop "running." With very simple assumptions one 
can generate standard-model-like hierarchies in the QFP's of the Yukawa couplings. Recent work on general questions regarding fixed points in supersymmetric theories [8] leads us to believe that a similar fixed point structure exists for the soft supersymmetry breaking terms.

However we also highlighted some difficulties with this picture. Most importantly, perturbation theory is only valid when the Yukawa couplings are already near their (lowscale) quasi-fixed values. Hence, the low scale QFP's are close to the perturbative limit and have, for example, the same status as gauge couplings in strong unification models $[9,10]$. (Indeed, from this analysis, it seems likely that any attempt to generate significant Yukawa hierarchies through the renormalization group will lead to a break down in perturbation theory.)

Beyond perturbation theory it is very difficult to say anything about the domain of attraction of these fixed points and hence it is not possible to determine to what extent hierarchical QFP's actually constitute a prediction. Despite this we find hierarchical QFP's intriguing and deserving of more study. A hint that further progress may be possible (which we shall not explore here) lies in the fact that, for the model we presented, the dominant pieces of the beta functions of the Yukawa couplings appear to have signs which alternate with order in $h^{2}$. This follows from the fact that, at any order, diagrams with Yukawa vertices dominate over those with gauge vertices, so that the beta function involves the same set of diagrams as the Wess-Zumino model. Sign alternation is characteristic of asymptotic series which are PadéBorel summable and indeed using this technique for the Wess-Zumino model shows that the domain of attraction of the QFP's are substantial [7]. Coupled with the properties of the underlying $N=2$ properties this may allow domains of attraction which are larger than the naive perturbative limit would imply.

\section{ACKNOWLEDGMENTS}

We are extremely grateful to Keith Dienes, Emilian Dudas, Tony Gherghetta, Laurent Lellouch, Joe Lykken and Riccardo Rattazzi for comments and conversations. S.F.K. would like to thank CERN and Fermilab for hospitality extended.
[1] I. Antoniadis, Phys. Lett. B 246, 377 (1990); I. Antoniadis, K. Benakli, and M. Quiros, ibid. 331, 313 (1994); J. D. Lykken, Phys. Rev. D 54, 3693 (1996); I. Antoniadis, S. Dimopoulos, and G. Dvali, Nucl. Phys. B516, 70 (1998); I. Antoniadis, N. Arkani-Hamed, S. Dimopoulos, and G. Dvali, Phys. Lett. B 436, 257 (1998).

[2] K. R. Dienes, E. Dudas, and T. Gherghetta, Phys. Lett. B 436, 55 (1998); hep-ph/9806292.

[3] G. Shiu and S-H. H. Tye, Phys. Rev. D 58, 106007 (1998); A. Pomarol and M. Quiros, Phys. Lett. B 438, 255 (1998); D. Ghilencea and G. G. Ross, hep-ph/9809217.

[4] M. Lanzagorta and G. G. Ross, Phys. Lett. B 349, 319 (1995).

[5] C. D. Froggatt and H. B. Nielsen, Nucl. Phys. B147, 277 (1979).

[6] B. Pendleton and G. G. Ross, Phys. Lett. 98B, 21 (1981); C. T. Hill, Phys. Rev. D 24, 691 (1981); C. T. Hill, C. N. Leung, and S. Rao, Nucl. Phys. B262, 517 (1985); W. A. Bardeen, M. Carena, S. Pokorski, and C. E. M. Wagner, Phys. Lett. B 320, 110 (1994); V. Barger, M. S. Berger, P. Ohmann, and R. J. N.
Phillips, ibid. 314, 351 (1993); V. Barger, M. S. Berger, and P. Ohmann, ibid. 314, 351 (1993); M. Lanzagorta and G. G. Ross, ibid. 364, 163 (1995); M. Carena and C. E. M. Wagner, Nucl. Phys. B452, 45 (1995); B. C. Allanach and S. F. King, ibid. B473, 3 (1996); B507, 91 (1997); Phys. Lett. B 407, 124 (1997); S. A. Abel and B. C. Allanach, ibid. 415, 371 (1997); 431, 339 (1998).

[7] P. M. Ferriera, I. Jack, and D. R. T. Jones, Phys. Lett. B 392, 376 (1997).

[8] I. Jack and D. R. T. Jones, hep-ph/9809250; S. F. King and G. G. Ross, Nucl. Phys. B530, 3 (1998).

[9] G. G. Ross, Phys. Lett. B 364, 216 (1995); D. Ghilencea, M. Lanzagorta, and G. G. Ross, ibid. 415, 253 (1997); Nucl. Phys. B511, 3 (1998); W. Pokorsski and G. G. Ross, ibid. B526, 81 (1995); King and Ross [8]; S. A. Abel and S. F. King, Phys. Lett. B 435, 73 (1998).

[10] C. Kolda and J. March-Russell, Phys. Rev. D 55, 4252 (1997); G. Amelino-Camelia, D. Ghilencea, and G. G. Ross, Nucl. Phys. B528, 35 (1998). 